\title{
IMAGE RETRIEVAL USING CERTAIN BLOCK BASED DIFFERENCE OF INVERSE PROBABILITY AND CERTAIN BLOCK BASED VARIATION OF LOCAL CORRELATION COEFFICIENTS INTEGRATED WITH WAVELET MOMENTS
}

\author{
${ }^{1}$ Thenkalvi, B. and ${ }^{2}$ S. Murugavalli \\ ${ }^{1}$ Sri Muthukumaran Institute of Technolgy, Anna University, Chennai, Tamilnadu, India \\ ${ }^{2}$ Panimalar Engineering College, Chennai, Tamilnadu, India
}

Received 2014-01-06; Revised 2014-02-14; Accepted 2014-04-03

\begin{abstract}
Content Based Image Retrieval (CBIR) is an evolving topic under image processing. Retrieval on medical images plays a vital role in saving mankind. Medical Content Based Image Retrieval (MCBIR) does not stop its work in displaying similar images; it also goes one step further for diagnosis to decide the method of therapy by comparing the query image and database image. Hence we believe that, our work is a mile stone in the medical imaging research and will gain an appreciable amount of demand in automatic diagnosis done through artificial intelligence. While handling a large amount of data base, retrieval naturally reduces the search area. Many research works were conducted on CBIR systems to yield better performance on retrieval of medical images. Here we propose a modified integrated approach which extracts low level image features: Color, intensity, shape and texture using Certain Block based Difference of Inverse Probability (CBDIP) and Certain Block based Variation of Local Correlation coefficients (CBVLC) for pre-processed images. Consequently wavelet moments are calculated on derived CBDIP and CBVLC values that leads to mining on medical images only with 48 feature vectors. Thereby greatly reducing total number of feature vectors used for similarity comparison for retrieval of similar images. To know the retrieval accuracy, precision and recall values are calculated for the mined 1000 images. It has been examined that this integrated approach shows an improvement in retrieval accuracy and the time taken for similarity comparison.
\end{abstract}

Keywords: CBCLC, CBIR, MCBIR, CBDIP, Wavelet

\section{INTRODUCTION}

Retrieval has its own past and present history from text based retrieval to Content Based Image Retrieval (CBIR). Text based retrieval was found to be frail on images due to its wrong annotation. This leads to the development of content based image retrieval i.e., retrieval based on contents of image: Low level features such as color, shape, intensity, texture. It is quite natural that the retrieval difficulty increases with respect to the increase in database size. Many CBIR systems were developed to search relevant images from the database.

CBIR system normally works with the following stages: Preprocessing, feature extraction and similarity matching. Corresponding Author: Thenkalvi, B., Sri Muthukumaran Institute of Technolgy, Anna University, Chennai, Tamilnadu, India
Precision and recall are the two metrics used to calculate retrieval accuracy. Preprocessing may involve image resizing, image enhancement or noise removal.

Resizing plays a predominant role in determining training and retrieval time. Histogram equalization is a widely used technique for enhancement whereas for the work based on medical images those changes are not required since medical images are acquired through standards. Since presence of noise may reduce the retrieval performance, removal of noise is mandatory in the case of geographic images/satellite images.

Once pre-processing completes, feature extraction is carried out to reduce the dimensionality of the data. The general architecture of CBIR system is shown in Fig. 1. 
Features extracted from the input query image and the images in the dataset are compared for retrieving relevant images. The most common method for comparing two images in content-based image retrieval is by calculating distance measure between the input image and images in the database using feature vectors stored in the feature database.

Many CBIR systems were developed which has its own significance. Color histogram is commonly used as a color descriptor for color feature extraction. Image clustering is used as a preprocessing step to overcome the problem of handling large dataset. Ranklet transform is used as a preprocessing step that generates 3 images with orientations vertical, horizontal and diagonal for each layer of RGB. Mean, standard deviation and skewness are calculated for the 3 orientations which are then used for similarity matching. Morphological color descriptors are used for color feature extraction on common images in the work proposed by Aptoula and Lefèvre (2009).

Kekre et al. (2010) proposed a Mask shape- Block Truncation Coding (BTC) Image Retrieval System. Here the low level image feature, shape is extracted from the image using Gradient operators and the same is used for similarity matching. The foresaid scheme is tested on 1000 generic images. Hemalatha et al. (2013) proposed a method for CBIR system using Discrete Cosine Transform (DCT). They concentrated on color and shape feature of an image. RGB model and DCT on segmented image are used to extract color and shape features. These feature vectors are cast-off for retrieving similar images.

Bencharef et al. (2013) used Color Histogram, Segmentation based Fractal Texture Analysis (SFTA) and Zernike moments to extract 3 low level image features color, shape and texture which acts as feature vectors for comparing database image and query image.

Low level image features are not only the factors behind image retrieval, but also human intervention was involved to build the semantic gap between high level and low level image features. Relevance Feedback mechanism is a widely used approach to overcome aforesaid point. To handle positive and negative feedback separately and unlabeled samples, Biased Maximum Margin Analysis (BMMA) was proposed by Zhang et al. (2012) which was named by them as Interactive Image Retrieval. From this, we infer that, computational time is high and it is applicable only for generic images. Lai and Chen (2011) introduced an Interactive Genetic Algorithm (IGA) based image retrieval technique where color and texture features are extracted by HSV color space, Gray Level Cooccurrence Matrix (GLCM) and Edge descriptors. Furthermore results are refined based on evaluation status obtained from user satisfaction test. For this kind of retrieval, human interaction ais required. Hamid (2011) introduced a CBIR system based on Color Layout Descriptor and Gabor Filter. Low level image features color and texture features are extracted utilizing the foresaid methods for similarity matching. Kishore et al. (2013) presented a study focused on sign language gesture recognition. Here image contents are extracted using Gabor filter and Chan Vese active Contour model for recognizing hand gesture of Indian sign language. Hiremath and Jagadeesh (2007) proposed a CBIR system based on Co-occurrence Histogram and Gradient Vector Flow field for extracting Color, Shape and Texture features for retrieval of similar images as a result of comparing query image and the dataset.

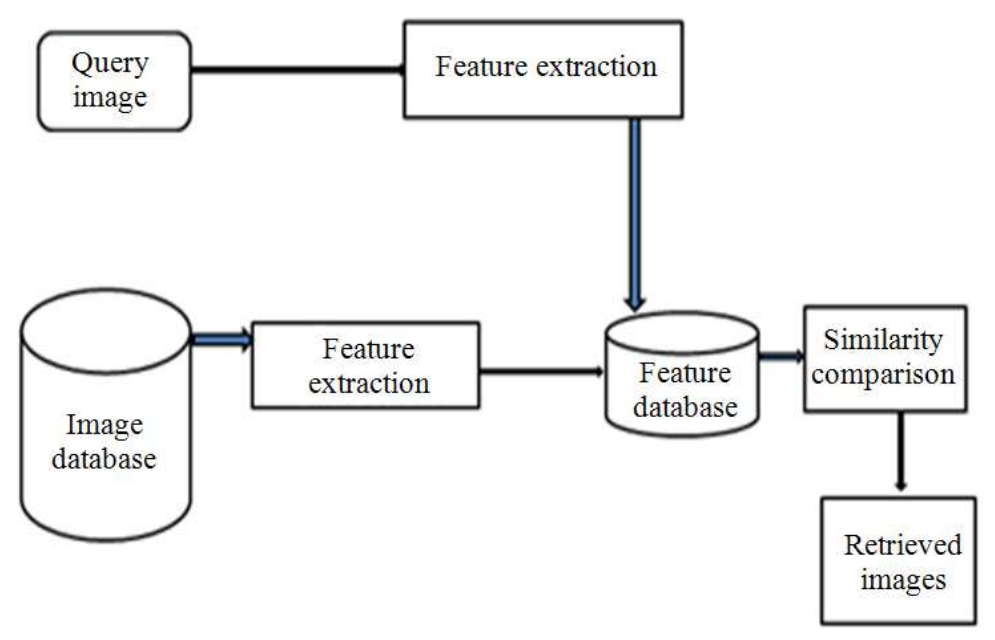

Fig. 1. Architecture of general CBIR system 
Hamid and Abdullah (2013) introduced an electromagnetism optimization technique based CBIR. This approach is implemented on 8000 images where each image is considered as an electrical charge. Here histogram and GLCM are used for feature extraction on resized images. It has been examined from the above system that the computational time is high. Therefore a good method is required to speed up the retrieval process.

Certain CBIR systems were implemented for medical images (Dimitris et al., 2009; Naqa et al., 2004; Kwak et al., 2002; Schnorrenberg et al., 2000; Zheng et al., 2003). Retrieval is performed on MRI brain images for the given 2D MR brain image using SVM based classification on the Datasets Brain Web and MIDAS. To evaluate the performance, False Match Rate (FMR) is calculated (Quddus and Basir, 2012). This is applicable only for MRI images. Gaussian Mixture Modelling Kullback-Leibler (GMM-KL) measure is used for image categorization and retrieval of 1500 radiological images (Greenspan and Pinhas, 2007). Support Vector Machine (SVM) is used as a classification technique on 5000 biomedical images where human interaction is involved through their feedback for retrieval of relevant images. For each recall, it showed 10 to $15 \%$ improvement in precision (Rahman et al., 2011). Dimitris et al. (2009) projected a Medical Image Retrieval system for radiographic images using patterns. Low level image features: Color, shape and texture are extracted. Then expectation maximization algorithm is applied for clustering. Generated patterns are compared with the patterns in the pattern database to retrieve the images that matches the query image.

Sriramakrishnan and Shanmugam (2012) proposed a method for medical image retrieval using fuzzy edge detection and segmentation algorithm where bell fuzzy multilayer neural network was constructed. The foresaid method produced $93.2 \%$ accuracy as a result of testing conducted on 44 images of 3 categories. From this we infer that the database size is very small. CBIR was exploited on Magnetic Resonance Image (MRI) brain images where the features are extracted using third order moment invariant functions. Binary association rules are used to organize and mark significant features of image database. Then the similarity comparison is performed through trigonometric function distance similarity measurement algorithm (Akila and Maheswari, 2013). From this study, it is understood that this method works well on MRI images. Certain works had been done related to diabetes diagnosis with the help of one dimentional data using Haar wavelets in Arangasamy et al. (2014), which reveals the importance of using Haar wavelets in our proposed work.
From the review on recent papers, it has been examined that certain works were performed only on particular type of images and specific features of image. Retrieval was forced on those set of images. As the nature of images increases in the database, complexity in retrieval process also increases proportionally. In this study, we focus CBIR using CBDIP, CBVLC and wavelet moments based on BDIP and BVLC defined by Chun et al. (2003).

\section{MATERIALS AND METHODS}

Figure 2 depicts the CBIR system for medical images. Heterogeneous images have been collected from various databases available in cyberspace and Scan Centre. Images taken for observation are 1000 medical images of following categories: 218 bone images, 415 mammogram images, 40 optical retinal images, 300 brain images and 27 retinal blood vessel images. The above system works with the following stages: Preprocessing, Feature Extraction, Similarity Comparison and Precision-Recall Calculation. As a first step, Dataset is preprocessed and then the image features are extracted using CBDIP and CBVLC. Discrete Wavelet transform is applied on the calculated CBDIP and CBVLC values through HAAR Wavelet. Then the derived features are stored in the database. Query image is preprocessed in the same way as the Dataset. The low level image Features captured from the query image is then compared with the feature vectors stored in the feature database. Similarity measure is computed using Euclidean distance measure to display the relevant images. Finally Precision and recall value is calculated to show the retrieval accuracy.

\subsection{Preprocessing}

Preprocessing is the first step in any image processing technique to handle noise, resize or enhance the image for further processing. Here we resize the image by considering the feature space of large sized images and to reduce the time spent on feature extraction and comparison. Images of different sizes are read by the matlab code and they are resized to $120 \times 120$ pixels. Importance of pre processing had been concentrated in the works done by Meenakshi and Anandhakumar (2013). Classification accuracy of about $2 \%$ had been obtained in brain tumor recognition. Calcagno et al. (2014), has mentioned the importance of removing the noise present in the image before it is processed for further feature extraction, which may increase the percentage of accuracy. 


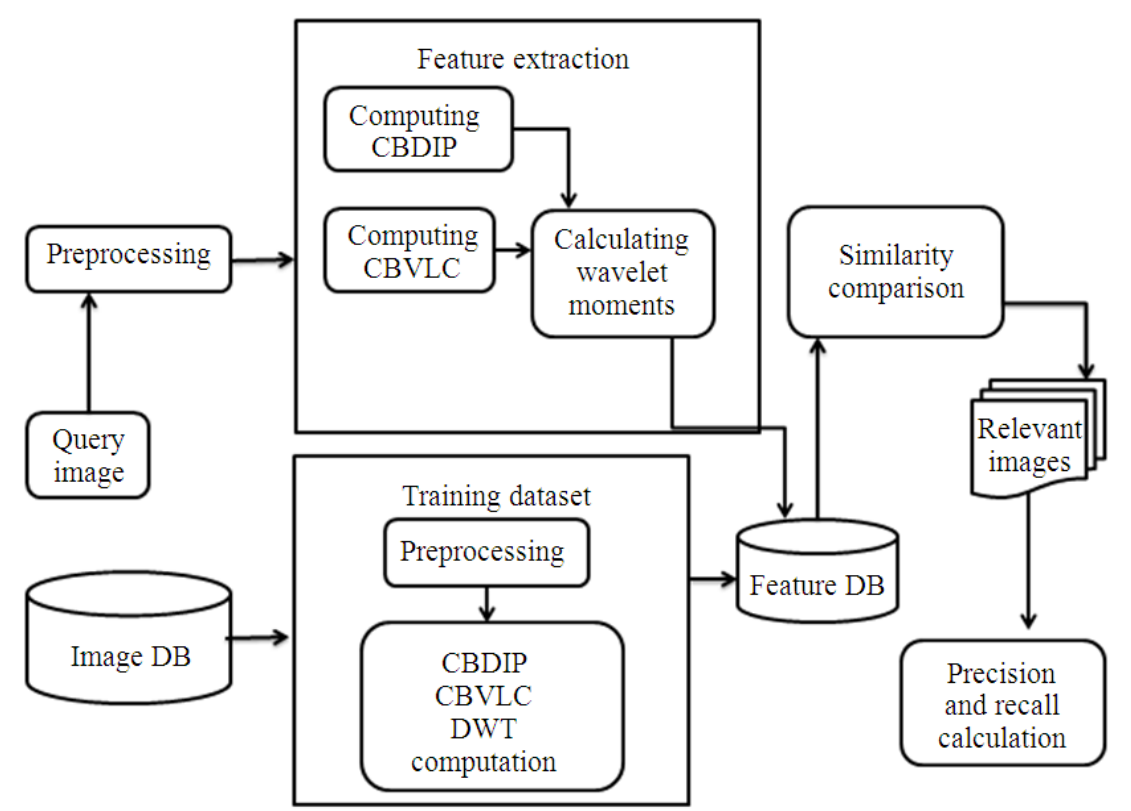

Fig. 2. Architecture of CBIR system using CBDIP, CBVLC and Wavelet Moments

\subsection{Feature Extraction}

This phase is used to extract low level image features such as Color, Intensity, Shape and Texture using the approaches Certain Block based Difference of Inverse Probability (CBDIP) and Certain Block based Variation of Local Correlation coefficients (CBVLC). BDIP and BVLC are the concepts referred from the work of Chun et al. (2003) and here they are renamed as CBDIP and CBVLC due to its property of handling uncertainty (Placing a non-zero value when the denominator becomes zero). Then Discrete Wavelet Transform (DWT) is applied on the derived CBDIP and CBVLC to extract reduced set of features.

\subsection{CBDIP}

CBDIP is a block based computational approach used in our CBIR system to extract color and intensity feature from the images. CBDIP is calculated as follows:

Step 1: Consider the preprocessed image of size $120 \times 120$ and divide the image into $n$ no of blocks. Each block (B) is of size $2 \times 2$.

Step 2: For each block B, Calculate BDIP which is defined as the difference between the number of pixels in a block and the ratio of the sum of pixel intensities in the block to the maximum in the block (Chun et al., 2003):

$$
\operatorname{BDIP}=M^{2}-\frac{\sum_{(i, j) \in B} I(I, j)}{\operatorname{Max}_{(i, j) \in B} I(i, j)}
$$

Step 3: Calculate skewness for each block of size $2 \times 2$ where skewness is given by:

Skewness $=\frac{M \times N}{(M \times N-1)(M \times N-2)} \sum_{x=0}^{M-1} \sum_{y=0}^{N-1}\left(\frac{I(i, j)-\bar{\mu}}{s t d}\right)^{3}$

In the Equation 1 and $2, I(i, j)$ denotes the intensity of a pixel $(i, j)$ and a block of size $M \times M$. The larger the variation of intensities is in a block, the higher the value of BDIP. When the highest intensity max I $(i, j)$ in a block is zero, then it may lead to get BDIP value as infinity and hence uncertainty arises. This is eliminated in our approach. Instead of zero, a nearly zero number is substituted in the formula to avoid the uncertainty; hence we call this as Certain BDIP (CBDIP). Here the skewness is added to detect the orientation of the gray levels as well.

Step 4: Calculate the final value of CBDIP as Equation 3:

$$
\mathrm{CBDIP}=\mathrm{BDIP} \times \text { Skewness }
$$

Hence CBDIP method becomes powerful one to catch the intensity variation and direction of variation as well by including skewness. 


\subsection{CBVLC}

CBVLC is also a block based approach to extract shape and texture smoothness. It is defined as the variation, or the difference between the maximum and minimum of local correlation coefficients according to six orientations. CBVLC is a block-based version of the VLCC (Chun et al., 2003) with 6 orientations. Each local correlation coefficient is defined as local covariance normalized by local variance.

CBVLC is calculated using the following steps

For each block B of size $2 \times 2$

Step1: Calculate Mean, Variance and Standard Deviation for both the original block and extended block (by considering the orientation).

Step2: Calculate correlation coefficient as:

$$
\rho(k, l)=\frac{\frac{1}{M^{2}} \sum_{(i, j) \in B} I(i, j) I(i+k, j+l)-\mu_{0,0}, \mu_{k, 1}}{\sigma_{0,0} \sigma_{k, 1}}
$$

where, B denotes a block of size MxM and $\mu_{0,0}$ and $\sigma_{0,0}$ denote the local mean and standard deviation of the block B, respectively. The notation $\rho(k, 1)$ denotes a pair of horizontal shift and vertical shift associated with the six orientations like $\left(-90^{\circ}, 0,45^{\circ},-45^{\circ}, 135^{\circ}, 225^{\circ}\right)$ as shown in Fig. 3. $\mu_{\mathrm{k}, 1}$ and $\sigma \mathrm{k}, 1$ represent the mean and standard deviation of the block shifted by $\rho(k, 1)$ respectively. Chun et al. (2003) considered four orientations for calculating BVLC, which suffers from lack of some image contents.
(a) $\rho(0,1)$
(b) $\rho(1,0)(c) \rho(1,1)$
(d) $\rho(1,-1)$
(e) $\rho(-1,-1)(f)$ $\rho(-1,1)$

Step 3: Calculate CBVLC using the following formula Equation 5:

$$
\text { CBVLC }=\max [\rho(k, 1)]-\min [\rho(k, 1)]
$$

For six orientations $\mathrm{O}_{6}=\{(0,1),(1,0),(1,1),(1,-1),(-1,-$ $1),(-, 1)\}$ where $\rho(k, 1)$ is correlation coefficient calculated in step 2 using Equation 4:

Step 4: Repeat the above steps for 'n' no of blocks.

A sample of CBDIP and CBVLC images along with the original image is shown in the Fig. 4.

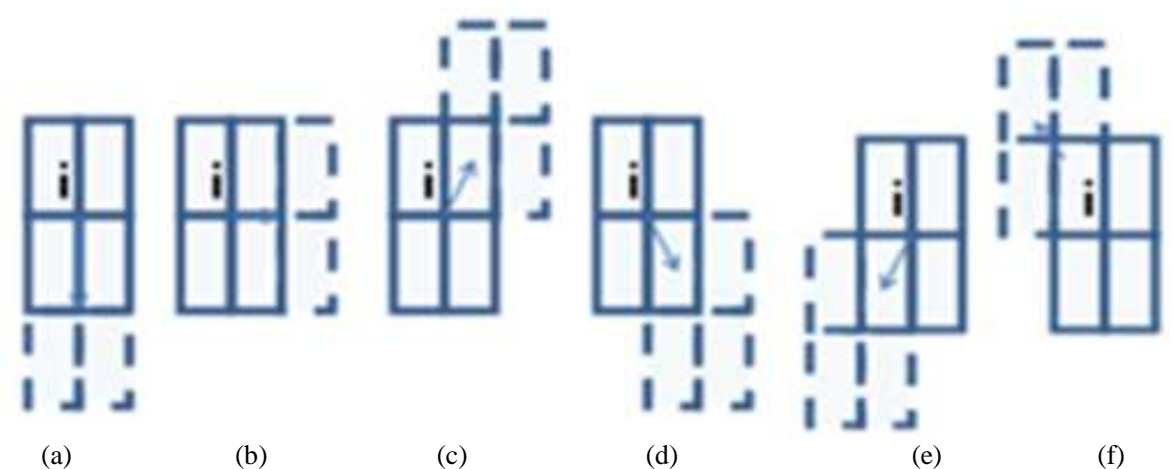

Fig. 3. Extended blocks based on six orientations

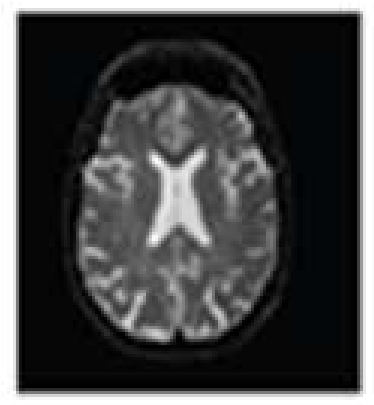

(a)

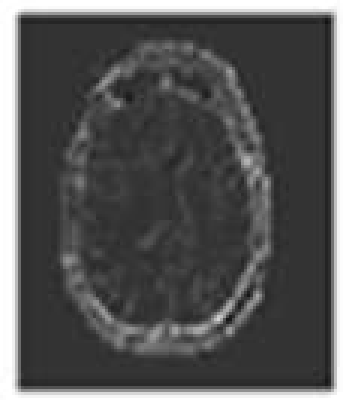

(b)

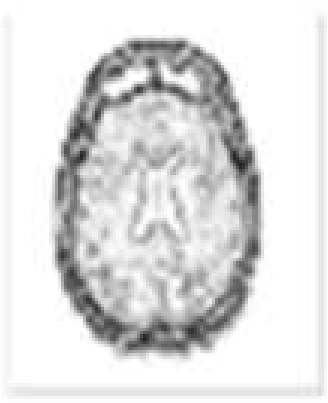

(c)

Fig. 4. Feature images (a) Original image (b) CBDIP image (c) CBVLC image 


\subsection{Discrete Wavelet Transform (DWT)}

Discrete Wavelet Transform (DWT) is applied on derived CBDIP and CBVLC. The simplest wavelet form among the wavelets, Haar wavelet is used here. Four sub bands of 3 level decomposition are shown in Fig. 5.

Figure 6 shows the DWT Process on CBDIP and CBVLC images for which steps are given below.

For each CBDIP and CBVLC Image:

Step 1: Decompose the image into 4 sub bands LL, LH, $\mathrm{HL}$ and $\mathrm{HH}$ using Haar Wavelet. As per the wavelet procedure, the original image fits into the LL sub band and the remaining sub bands acts as detail matrices.

Step 2: Calculate Mean $(\mu)$ and Standard Deviation $(\sigma)$ for the 4 sub bands as Equation 6 and 7:

$\mu_{\mathrm{m}}^{\mathrm{n}}=\frac{1}{\mathrm{~N}_{\mathrm{mn}}} \sum_{(\mathrm{i}, \mathrm{j}) \in \mathrm{W}_{\mathrm{m}}^{\mathrm{n}}} \mathrm{W}_{\mathrm{m}}^{\mathrm{n}}(\mathrm{i}, \mathrm{j})$

$\sigma_{\mathrm{m}}^{\mathrm{n}}=\sqrt{\frac{1}{\mathrm{~N}_{\mathrm{mn}}} \sum_{(\mathrm{i}, \mathrm{j}) \in \mathrm{W}_{\mathrm{m}}^{\mathrm{n}}}\left(\mathrm{W}_{\mathrm{m}}^{\mathrm{n}}(\mathrm{i}, \mathrm{j})-\mu_{\mathrm{m}}^{\mathrm{n}}\right)^{2}}$

where, $\mathrm{n}$ and $\mathrm{m}$ denote decomposition level and sub band orientation respectively, $\mathrm{N}_{\mathrm{mn}}$ is the number of coefficient in the $\mathrm{mn}_{\mathrm{th}}$ sub band, $\mathrm{W}_{\mathrm{m}}^{\mathrm{n}}(\mathrm{i}, \mathrm{j})$ the intensity of a pixel $(i, j)$ in the sub band image $W_{m}^{n}$ and $\mu_{m}^{n}$ and $\sigma_{\mathrm{m}}^{\mathrm{n}}$ are the mean and standard deviation of absolute values of coefficients in the $\mathrm{mn}_{\mathrm{th}}$ sub band respectively:
Step 3: Repeat step 1 and step 2 till the decomposition level reaches 3. (At each level, decomposition takes places only on LL sub band).

For level 1 decomposition, three high band images $\mathrm{W}^{1} \mathrm{HL}(\mathrm{i}, \mathrm{j}), \quad \mathrm{W}^{1} \mathrm{LH}(\mathrm{i}, \mathrm{j})$ and $\mathrm{W}^{1} \mathrm{HH}(\mathrm{i}, \mathrm{j})$ denotes horizontal, vertical and diagonal orientations respectively and the original image fits into $W^{1} L L(i, j)$. Similarly sub bands are depicted at each level of decomposition.

\subsection{Similarity Comparison}

This phase of our work is to compare feature vectors of query image and feature vectors of data base images and it is shown in Fig. 7. In our approach, number of feature vectors to be compared is greatly reduced to 48 . Euclidean distance measure is used to calculate the distance. Threshold is set as 0 to 0.1 to retrieve the relevant images based on the distance measure.

\subsection{Precision and Recall Calculation}

Precision and recall are the two metrics commonly used for calculating retrieval accuracy and they are calculated as:

- Precision $=$ Number of relevant images retrieved/Total number of images retrieved from data base

- Recall = Number of relevant image retrieved/Total number of relevant image in the data base

\begin{tabular}{|c|c|c|c|}
\hline $\mathrm{LL}_{3}$ & $\mathrm{HL}_{3}$ & \multirow{2}{*}{$\mathrm{HL}_{2}$} & \\
\cline { 1 - 1 } $\mathrm{LH}_{3}$ & $\mathrm{HH}_{3}$ & \\
\hline \multicolumn{2}{|c|}{$\mathrm{LH}_{2}$} & $\mathrm{HH}_{2}$ & \\
\hline & $\mathrm{HL}_{1}$ \\
\multicolumn{2}{|c|}{$\mathrm{LH}_{2}$} & \\
& & $\mathrm{HH}_{2}$ \\
\hline
\end{tabular}

Fig. 5. 3 Level decomposition 


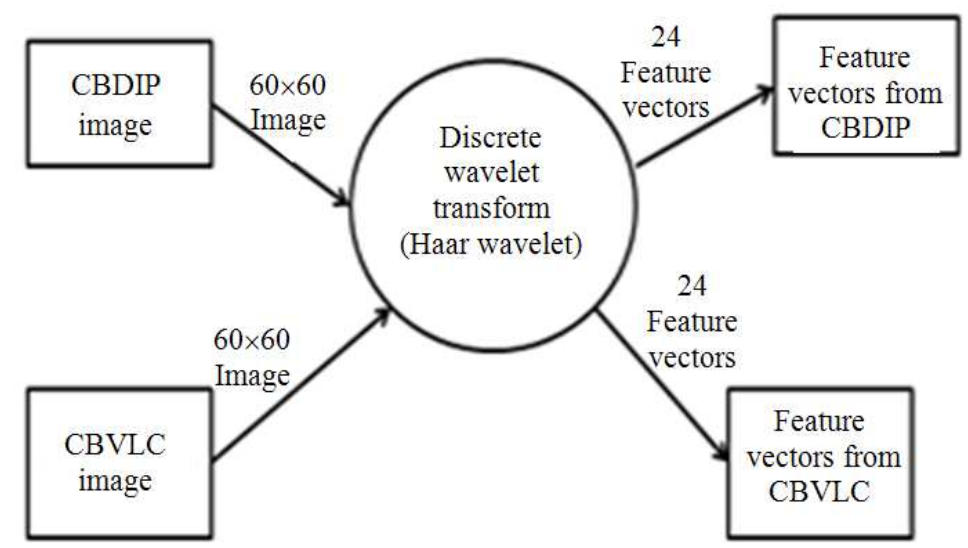

Fig. 6. DFD for DWT on CBDIP and CBVLC

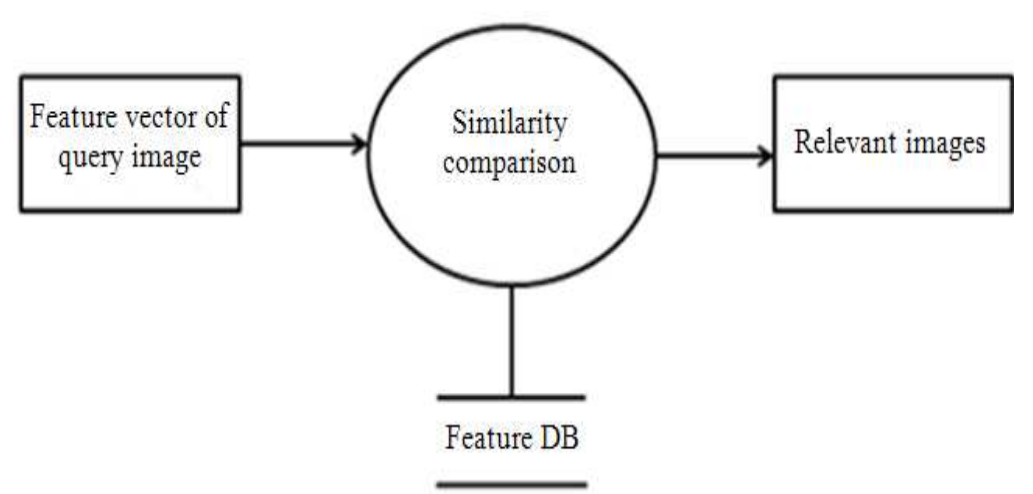

Fig. 7. DFD for similarity comparison between query image and dataset

\section{RESULTS}

The CBIR system discussed here was implemented using Matlab 2012a on Intel P4 2.2GHz, 4 GB memory and Windows 7 operating system. Each image in the database is considered as a query image to retrieve similar images. After Preprocessing, images are resized to $120 \times 120$. Resized image is given as input to the next stage, feature extraction. CBDIP and CBVLC are used to extract features. As a result, we get reduced image of size $60 \times 60$. Then DWT is applied on $60 \times 60$ CBDIP and CBVLC Image. Haar Wavelet on the given input (CBDIP and CBVLC Image) produced 48 feature vectors for 3 levels of decomposition. i.e., 24 feature vectors for each image. Features extracted from the query image are then compared with the features stored in the feature database created as a result of training the image database. A total of 1000 images were taken to verify the accuracy of the proposed system. 48 feature vectors are used to retrieve relevant images. Figure 8 shows the top 5 retrieved images for the given query image where the first column depicts query image and the remaining column shows retrieved images. In previous literatures, an average accuracy of $84 \%$ and even more had obtained for natural images with 96 feature vectors . In our proposed work, our major concern is to retrieve the medical images with less number of features.

\section{DISCUSSION}

The precision and recall curves obtained are as follows. Figure 9 shows the relationship between precision and recall. From Fig. 10, it is understood that, CBDIP+CBVLC moments outperforms in precision scores with 48 feature vectors (reduced set of feature vectors) when compared to BDIP+BVLC moments based on 96 features. The major claim in our work is that, the feature size is reduced by a factor of two thereby reducing the time taken for comparing the query image and dataset. 
Thenkalvi, B. and S. Murugavalli / Journal of Computer Science 10 (8): 1497-1507, 2014
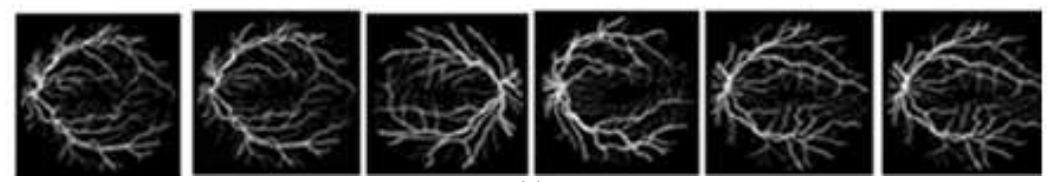

(a)
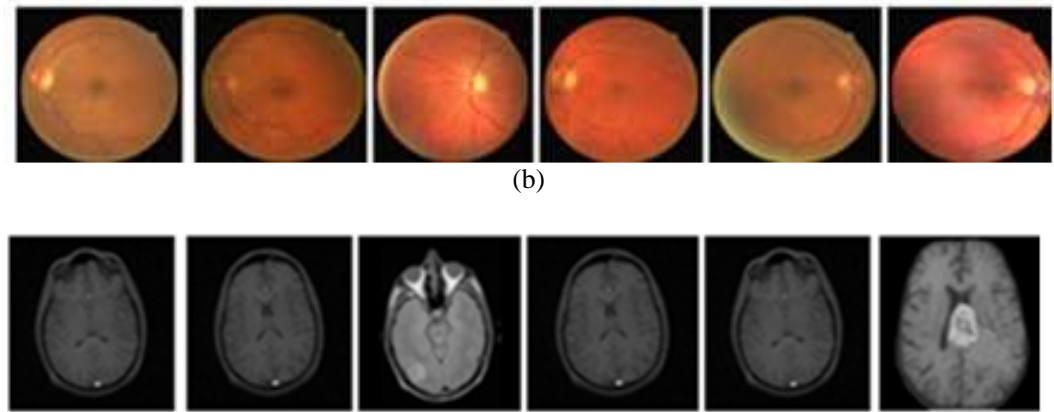

(b)
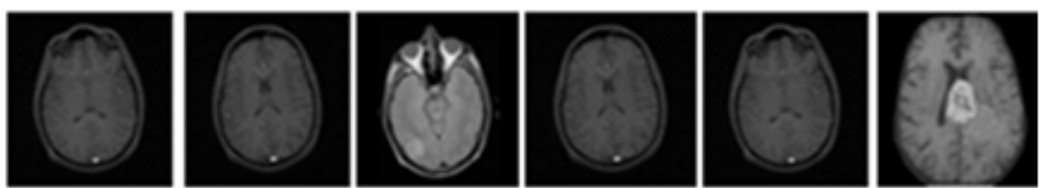

(c)
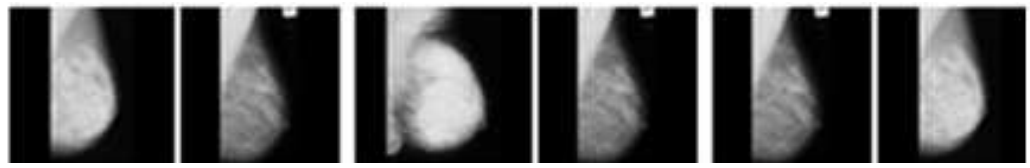

(d)

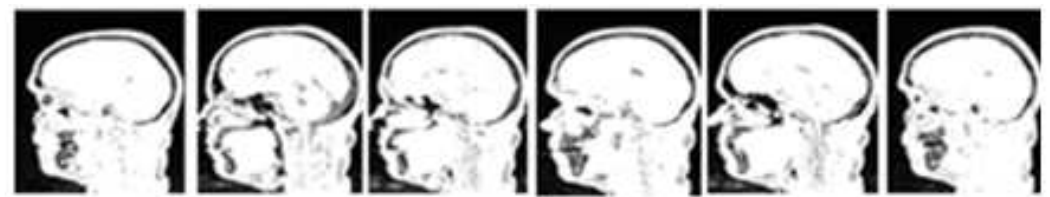

(e)

Fig. 8. (a) Retinal blood vessels (b) Retinal fundus images (c) Brain MRI (d) Mammogram images (e) Skull MRI

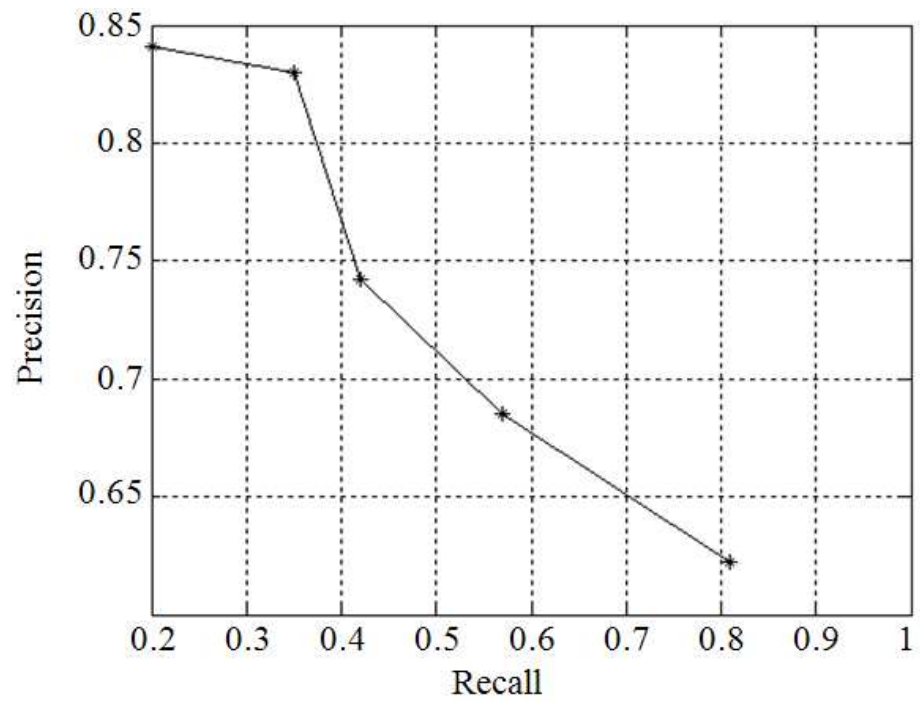

Fig. 9. Precision Vs. recall 
Thenkalvi, B. and S. Murugavalli / Journal of Computer Science 10 (8): 1497-1507, 2014

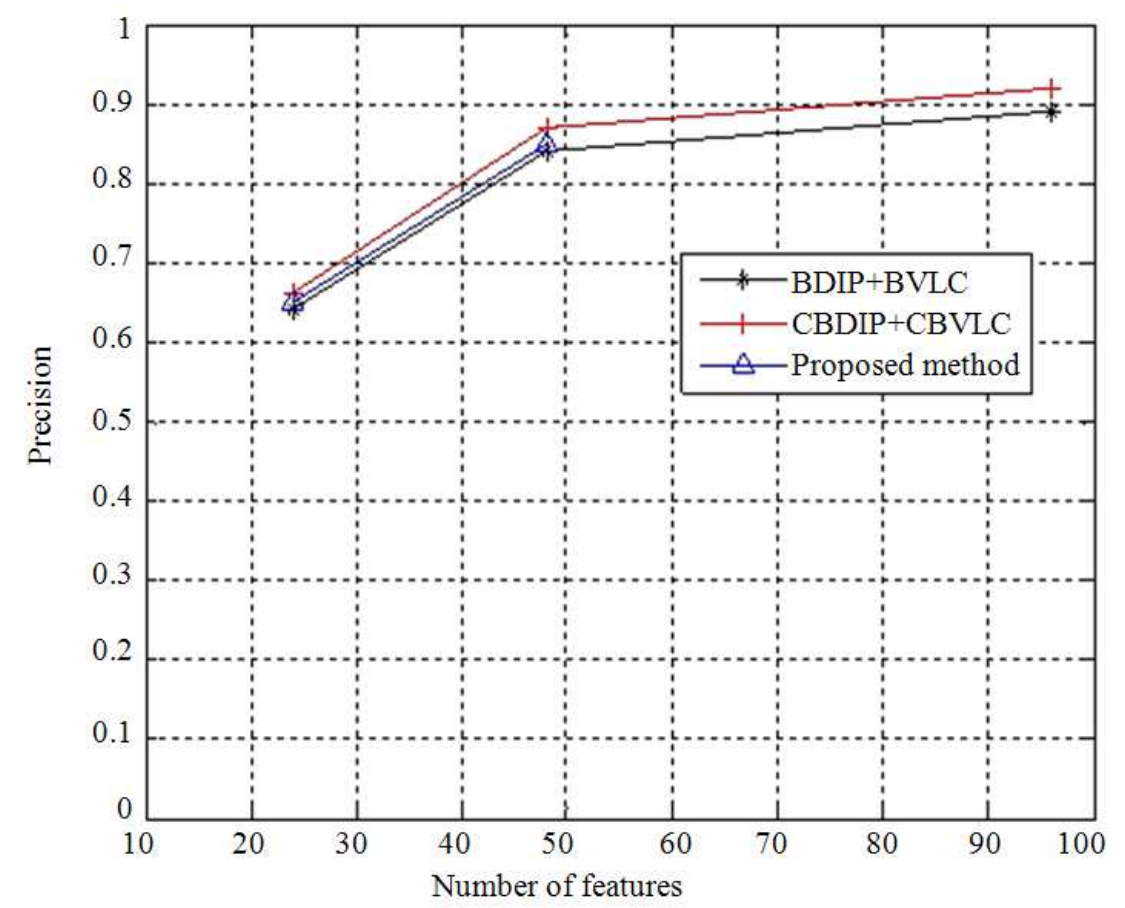

Fig. 10. Precision Vs. features

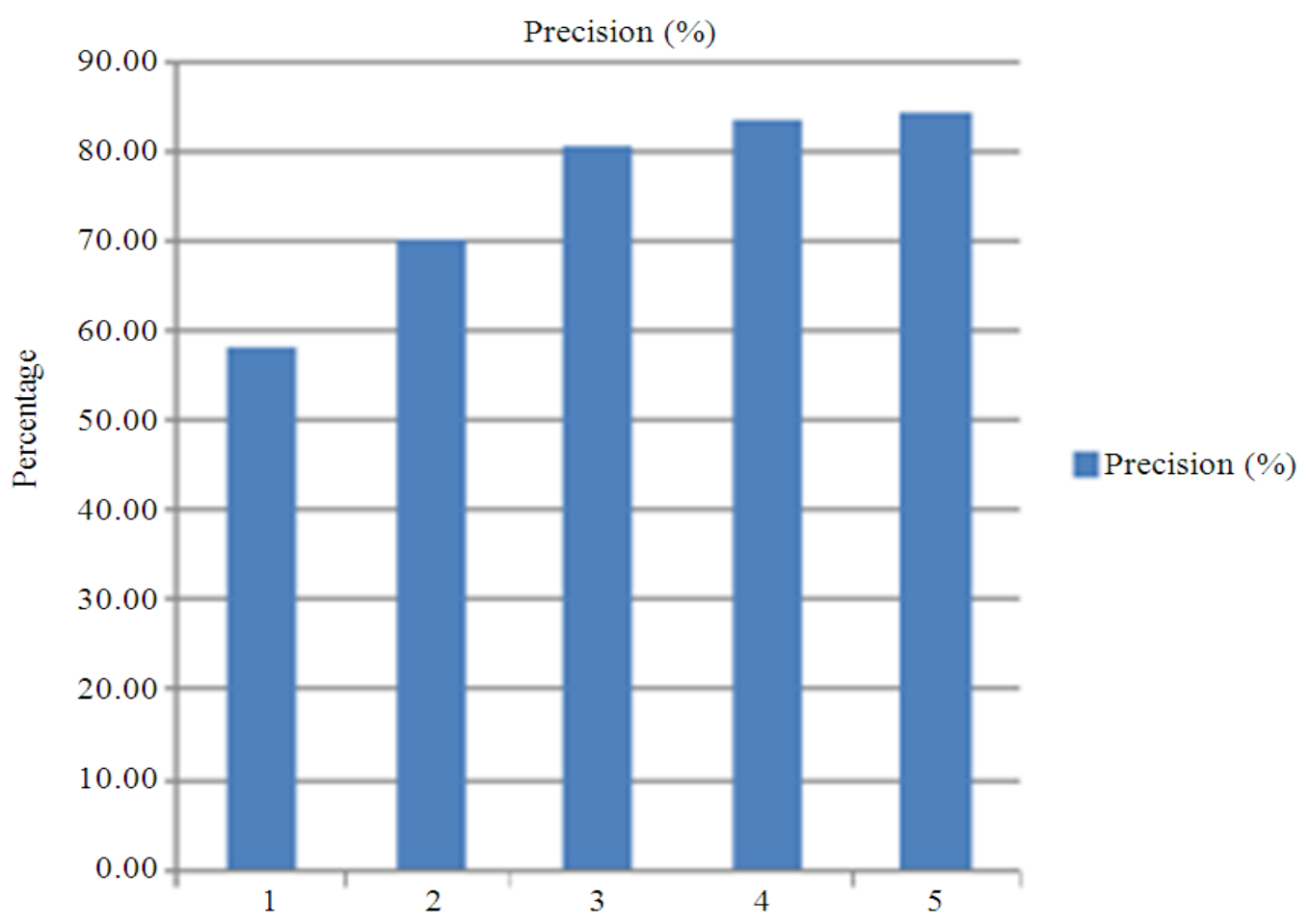

Fig. 11. Performance chart of CBIR systems based on precision value 


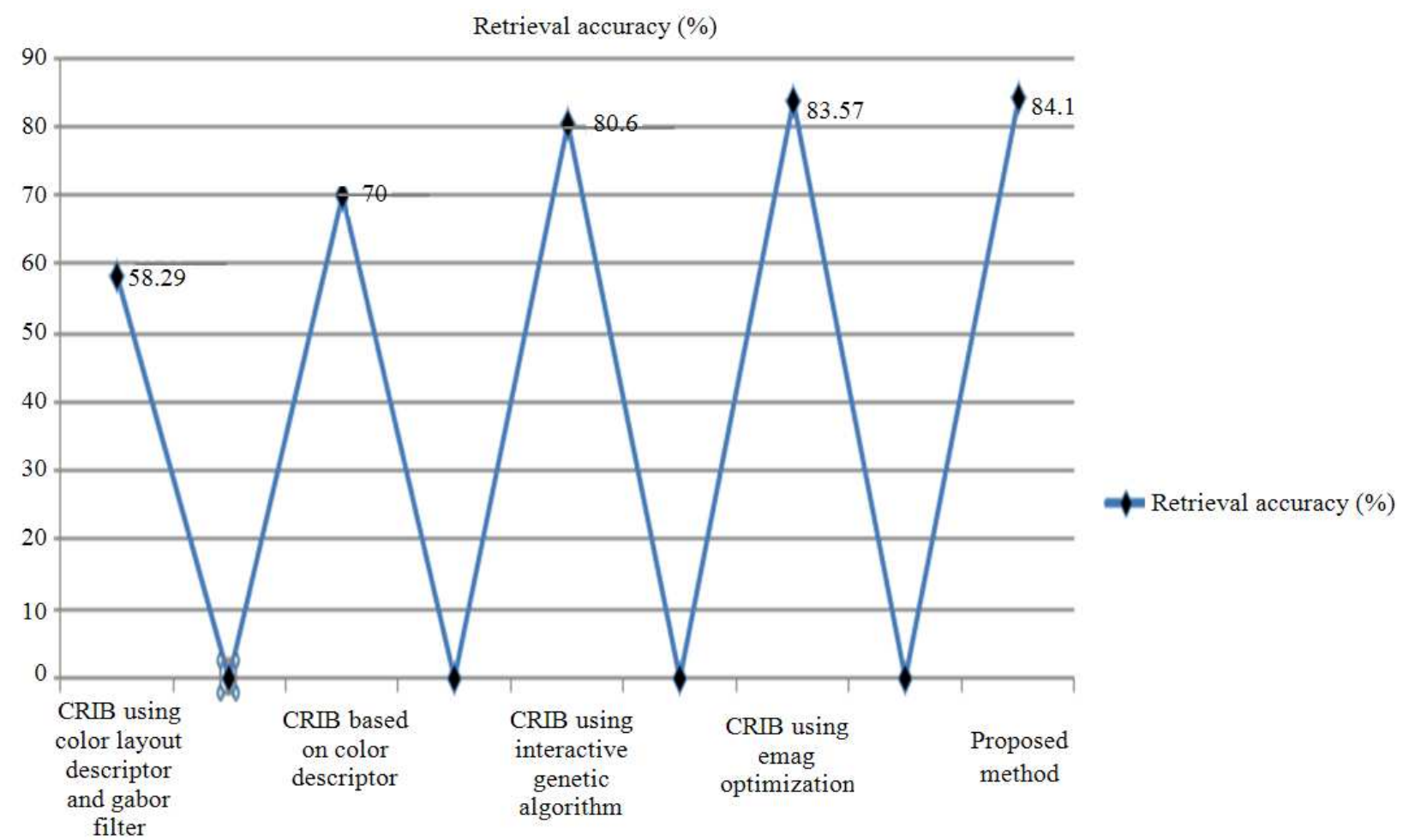

Fig.12. Comparison between proposed method and other works

Few CBIR systems are compared with our proposed methodology as shown in Fig. 11 and 12. Figure 11 is a bar representation of average precision obtained through various methods. Indexes from 1 to 5 in $x$ axis represent the methods as given in Fig. 12. Which is a line chart showing the comparison between proposed method and other works where retrieval accuracy is shown in percentage.

\section{CONCLUSION}

In this study, we have proposed a modified approach for content based image retrieval by making use of the concepts CBDIP, CBVLC and DWT. This proposed scheme is smeared on 1000 images where the image size is reduced stage by stage thereby reducing the feature vectors required for similarity comparison. The modified approach towards CBIR System shows an improvement of $1 \%$, but saves enormous time by reducing the number of feature vectors. In our future work, we will be considering large dataset for training and testing the retrieval accuracy. In real time medical image processing, there exists a very huge number of images approaching to infinity. Hence, the future work in the related topic is mainly to accommodate more images from various medical challenges, so as to reduce the diagnosis burden.

\section{REFERENCES}

Akila, T. and P.U. Maheswari, 2013. Robust magneticresonance brain image recognition using similarity measurement. J. Comput. Sci., 9: 678-689. DOI: $10.3844 /$ jcssp.2013.678.689

Aptoula, E. and S. Lefèvre, 2009. Morphological description color images for content-based image retrieval. IEEE Trans. Imag. Proc., 18: 2505-2517. DOI: $10.1109 /$ TIP.2009.2027363

Arangasamy, R., J. Sundararajan, I. Vennila and G. Shankar, 2014. Automatic glucose insulin regulation system-comparison of embedded control design and Harr wavelet method for type-1 diabetes. Am. J. Applied Sci., 11: 433-447. DOI: 10.3844/ajassp.2014.433.447

Bencharef, O., B. Jarmouni and A. Souissi, 2013. Research similar images based global descriptors multiple clustering. Int. J. Eng. Technol., 5: 3142-3151. 
Calcagno, S., F.L. Foresta and M. Versaci, 2014. Independent component analysis and discrete wavelet transform for artifact removal in biomedical signal processing. Am. J. Applied Sci., 11: 57-68. DOI: 10.3844/ajassp.2014.57.68

Chun, Y.D., S.Y. Seo and N.C. Kim, 2003. Image retrieval using BDIP and BVLC moments. IEEE Trans. Circuits Syst. Video Technol., 9: 951-957. DOI: 10.1109/TCSVT.2003.816507

Dimitris, K., I.N. Pelekis, E.E. Kotsifakos, I. Kopanakis and H. Karanikas et al., 2009. A pattern similarity scheme for medical image retrieval. IEEE Trans. Inf. Technol. Biomed., 13: 442-450. DOI: 10.1109/TITB.2008.923144

Greenspan, H. and T.P. Pinhas, 2007. Medical image categorization and retrieval for PACS using the GMM-KL framework. IEEE Trans. Inf. Technol. Biomedi., 11: 190-202. DOI: 10.1109/TITB.2006.874191

Hamid, A.J. and N.A. Abdullah, 2013. Content-based image retrieval based on electromagnetism-like mechanism mathematical problems in engineering.,

Hamid, A.J., 2011. Image retrieval system based on color layout descriptor and Gabor filters. Proceedings of the IEEE Conference Open Systems, Sep. 25-28, IEEE Xplore Press, Langkawi, pp: 3236. DOI: $10.1109 /$ ICOS.2011.6079266

Hemalatha, P.A., K.S. Ravichandran, B. santhi, 2013. Query by Image content using discrete cosine transform. Int. J. Eng. Technol.

Hiremath, P.S. and P. Jagadeesh, 2007. Content based image retrieval based on color, texture and shape features using Image and its comple-ment. Int. J. Comput. Sci. Security.

Kekre, H.B., S. Thepade, P. Mukherjee, M. Kakaiya and S. Wadhwa et al., 2010. Image retrieval with shape features extracted using gradient operators and slope magnitude technique with BTC. Int. J. Comput. Applic.

Kishore, P.V.V., S.R.C. Kishore and M.V.D. Prasad, 2013. Conglomeration hand shapes and texture information for recognizing gestures Indian sign language using feed forward neural networks. Int. J. Eng. Technol.,
Kwak, D.M., B.S. Kim, O.K. Yoon, C.H. Park and J.U. Won et al., 2002. Content-based ultrasound image retrieval using a coarse to fine approach. Ann. NY Acad. Sci., 980: 212-224. DOI: 10.1111/j.17496632.2002.tb04898.x

Lai, C.C. and Y.C. Chen, 2011. A user-oriented image retrieval system based on interactive genetic algorithm. IEEE Trans. Instru. Measure., 60: 33183325. DOI: 10.1109/TIM.2011.2135010

Meenakshi, R. and P. Anandhakumar, 2013. Wavelet statistical texture features with orthogonal operators tumour classification in magnetic resonance imaging brain. Am. J. Applied Sci., 10: 1154-1159. DOI: 10.3844/ajassp.2013.1154.1159

Naqa, E.I., Y. Yang, N.P. Galatsanos, R.M. Nishikawa and M.N. Wernick, 2004. A similarity learning approach to content-based image retrieval: Application to digital mammography. IEEE Trans. Med. Imag., 10: 1233-1244. DOI: 10.1109/TMI.2004.834601

Rahman, M.M., K. S.K. Antani and R.G. Thoma, 2011. A learning-based similarity fusion filtering approach for biomedical image retrieval using SVM classification relevance feedback. IEEE Trans. Inf. Technol. Biomedi.,

Schnorrenberg, F., C.S. Pattichis, C.N. Schizas and K. Kyriacou, 2000. Content-based retrieval of breast cancer biopsy slides Technol. Health Care., 8: 291-297.

Sriramakrishnan, C. and A. Shanmugam, 2012. An fuzzy neural approach for medical image retrieval. J. Comput. Sci., 11: 1809-1813. DOI: 10.3844/jessp.2012.1809.1813

Quddus, A. and O. Basir, 2012. Semantic image retrieval in magnetic resonance brain volumes. IEEE Trans. Inf. Technol. Biomedi., 16: 348-355. DOI: 10.1109/TITB.2012.2189439

Zhang, L., L. Wang and W. Lin, 2012. Semisupervised biased maximum margin analysis for interactive image retrieval. IEEE Trans. Imag. Processing.,

Zheng, A.W.W., J. Gilbertson and M.J. Becich, 2003. Design analysis content-based pathology image retrieval system. IEEE Trans. Inf. Technol. Biomed., 4: 249-255. 